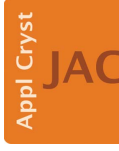

JOURNAL OF

APPLIED

CRYSTALLOGRAPHY

ISSN 1600-5767

Received 13 August 2018

Accepted 8 May 2019

Edited by A. J. Allen, National Institute of Standards and Technology, Gaithersburg, USA

Keywords: nanoparticles; diffraction; lattice parameters; accuracy; precision.

\section{Comment on the article The nanodiffraction problem}

\author{
Zbigniew Kaszkur*
}

Institute of Physical Chemistry, PAS, Kasprzaka 44/52, 01-224, Warszawa, Poland. *Correspondence e-mail: zkaszkur@ichf.edu.pl

The article The nanodiffraction problem (Xiong et al., 2018) discusses the origin of the peak shifts for nanocrystalline fragments of a perfect periodic lattice from their positions determined by Bragg's law. We submit that the article fails, however, in correct analysis of this phenomenon. It attempts to compare the peak shift from a thin-film pattern with the peak shift measured from a powder pattern (see, for example, the end of Section 5). The patterns are, however, not comparable owing to their different measurement geometry. The Debye formula describes the measured diffracted intensity. The alternative approach via Bragg peak description involves structure factors and the Lorentz factor $1 / \sin (2 \theta)$, normalizing the intensities over powder diffraction cones. This is not the case in equations $(3 b)$ and $(4 b)$, where spatial averaging is done at the level of interatomic distances. If the Debye formula and crystallographic formulae of the kind presented for the thinfilm slab in Section 7 [equations (6), (7) and (8)] are to be compared at the structure factor level, one has to assume that the Lorentz factor is inherently hidden within the Debye formula. So the correct comparison should be made between the thin-film case and the Debye formula multiplied by $\sin (2 \theta)$. The results obtained by Xiong et al. (2018) can be explained if we assume that the Lorentz correction is present within the Debye approach.

The peak shift caused by the Lorentz factor can be treated in the same approximate way as the shift caused by the $1 / \sin (\theta)$ correction considered by the authors in Section 7.2. Approximation of the diffraction peak close to its maximum by a Gaussian function allows us to estimate the peak shift analytically. Careful analysis reveals, however, that the relation of the Gaussian dispersion parameter $\sigma$ to the FWHM $\beta$ [below formula (14)] lacks the factor of 2 arising because the FWHM appearing in the Scherrer formula has to be measured in $2 \theta$. So the correct relation is $\beta_{2 \theta}=4 \sigma[2 \ln (2)]^{1 / 2}$. In this estimation the $B$ parameter has to be given by $B=2 / \tan \left(2 \theta_{\mathrm{B}}\right)$. The final $\Delta a / a=4 / \tan \left(\theta_{\mathrm{B}}\right) B \sigma^{2}$ can be expressed by the crystal size from the Scherrer equation as

$$
\Delta a / a=\left(C^{2} d_{h k l}^{2}\right) /\left[2 \ln (2) D^{2}\right]\left[1-\tan ^{2}\left(\theta_{\mathrm{B}}\right)\right],
$$

where $C$ is the Scherrer constant, $D$ is the crystal size and $d_{h k l}$ is the interplanar distance for the considered $h k l$ peak.

Although the thin film can cause a size-dependent shift of the peaks from their Bragg positions that decreases with angle with the same rate as the decrease of the square of the interatomic distances, for powder diffraction we have two sources of shift. The second, arising from the Lorentz factor, shifts the diffraction peak already displaced owing to powder nanocrystallinity (intrinsic effect) further by a value that is comparable to the thin-film case, but modified by the $1-\tan ^{2}(\theta)$ factor. For angles $\theta$ larger than $45^{\circ}$, the whole correction becomes negative. This is clearly visible in Fig. 5 of Xiong et al. (2018), where the point of crossing zero for each pattern calculated for three wavelengths corresponds to the $q$ value calculated for $45^{\circ}$. Similarly, the assumption that the Lorentz correction is inherently hidden within the Debye equation explains Fig. 6, where all $\Delta a / a$ lines cross zero at $2 \theta=90^{\circ}$. The non-monotonic changes of the shift (ups and downs) are repeatable, which suggests that the accuracy of the fit is quite good. The effect of the peak overlap that the authors were concerned about is an unavoidable feature of nanocrystalline diffraction patterns, and the 'absolute' peak position does not exist.

Most of the 'extended analysis' of the powder diffraction results offered by the authors does not touch the core of the problem because they do not separate the angular correction shifts from the intrinsic ones. As was shown above, these intrinsic shifts result 
from Debye summation and are characteristic for nanopowder diffraction - they are not present in the thin-slab case considered by Xiong et al. (2018). These intrinsic shifts violating Bragg's law are a function of crystalline structure (its radial distribution function) and merit closer examination. For real nanocrystals the problem is more complex but can be approached by experiment (Kaszkur, 2000a,b, 2001, 2004; Kaszkur et al., 2005, 2006, 2015, 2017; Kaszkur, Rzeszotarski \& Juszczyk, 2014; Kaszkur, Mierzwa et al., 2014; Rzeszotarski \& Kaszkur, 2009).

\section{References}

Kaszkur, Z. (2000a). J. Appl. Cryst. 33, 1262-1270.

Kaszkur, Z. (2000b). J. Appl. Cryst. 33, 87-94.
Kaszkur, Z. (2001). Mater. Sci. Forum, 378-381, 314-319.

Kaszkur, Z. (2004). Phys. Chem. Chem. Phys. 6, 193-199.

Kaszkur, Z. (2006). Z. Kristallogr. Suppl. 2006, 147-154.

Kaszkur, Z., Juszczyk, W. \& Łomot, D. (2015). Phys. Chem. Chem. Phys. 17, 28250-28255.

Kaszkur, Z., Mierzwa, B., Juszczyk, W., Rzeszotarski, P. \& Łomot, D. (2014). RSC Adv. 4, 14758-14765.

Kaszkur, Z., Mierzwa, B. \& Pielaszek, J. (2005). J. Appl. Cryst. 38, 266-273.

Kaszkur, Z., Rzeszotarski, P. \& Juszczyk, W. (2014). J. Appl. Cryst. 47, 2069-2077.

Kaszkur, Z., Zieliński, M. \& Juszczyk, W. (2017). J. Appl. Cryst. 50, 585-593.

Rzeszotarski, P. \& Kaszkur, Z. (2009). Phys. Chem. Chem. Phys. 11, 5416-5421.

Xiong, S., Öztürk, H., Lee, S.-Y., Mooney, P. M. \& Noyan, I. C. (2018). J. Appl. Cryst. 51, 1102-1115. 


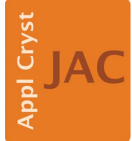

JOURNAL OF

APPLIED

CRYSTALLOGRAPHY

ISSN 1600-5767

Received 4 September 2018

Accepted 8 May 2019

Edited by A. J. Allen, National Institute of Standards and Technology, Gaithersburg, USA

Keywords: nanoparticles; diffraction; lattice parameters; accuracy; precision.

\section{Response to Zbigniew Kaszkur's comment on the article The nanodiffraction problem}

\author{
Shangmin Xiong, ${ }^{a}$ Hande Öztürk, ${ }^{b}$ Seung-Yub Lee, ${ }^{a}$ Patricia M. Mooney ${ }^{a, c}$ and \\ Ismail Cevdet Noyan ${ }^{\mathrm{a} *}$
}

a Applied Physics and Applied Mathematics, Columbia University, New York, NY 10027, USA, b National Synchrotron Light Source II, Brookhaven National Laboratory, Upton, NY 11973, USA, and ${ }^{\mathrm{c}}$ Department of Physics, Simon Fraser University, Burnaby, BC V5A 1S6, Canada. *Correspondence e-mail: icn2@columbia.edu

In a previous article (Öztürk et al., 2015) we showed that the classical Lorenz factor $\left(L_{\mathrm{F}}=\right.$ $1 / \cos \theta)$ and its extensions are inapplicable for correcting diffraction patterns from nanoparticles with diameters, $D$, smaller than $20 \mathrm{~nm}$. However, Kaszkur (2019) suggests that in our article The nanodiffraction problem (Xiong et al., 2018) 'the correct comparison should be made between the thin-film case and the Debye formula multiplied by $\sin (2 \theta)$ '. This factor, termed the 'single-crystal Lorenz factor', accounts for the change in the irradiated volume of the crystal as a function of $2 \theta$ (Reynolds, 1986). Since the diffraction patterns in our previous article were simulated assuming an infinite number of crystallites in the powder diffraction analysis, and an infinitely large slab of finite thickness irradiated by a plane wave for the thin-film case, we do not consider such a correction theoretically justified. Nevertheless, we tested Kaszkur's hypothesis using numerical simulations. Fig. 1(a) shows the expected diffraction pattern for a monodisperse powder sample consisting of infinitely many, ideal, Au spheroids, $5 \mathrm{~nm}$ in diameter, computed using the modified Debye formalism (https://github.com/wojdyr/ debyer) with $\mathrm{Cr} K \alpha$ radiation. The variation of the suggested correction factor, $C_{\mathrm{F}}=$ $\sin (2 \theta)$, over this angular range and the corrected intensity profile obtained by multiplying the Debye intensity profile with this factor are also plotted. In Fig. 1(b) the lattice parameter errors, $\Delta a_{h k l}=a_{h k l}-a_{\text {ideal }}$, computed from the individual peak positions for these two profiles are shown. We do not see any improvement over the uncorrected results. We observed similar results for (corrected) patterns computed using other wavelengths and for full-pattern fitting. For larger-diameter $(D>20 \mathrm{~nm})$ particles, we recovered the original lattice parameter without using any corrections. We conclude that (i) applying the 'Lorenz correction' to computed nanoparticle powder patterns requires further study and (ii) the hypothesis put forward by Kaszkur (2019) cannot be used to correct the patterns reported in our current article (Xiong et al., 2018). We hope that our

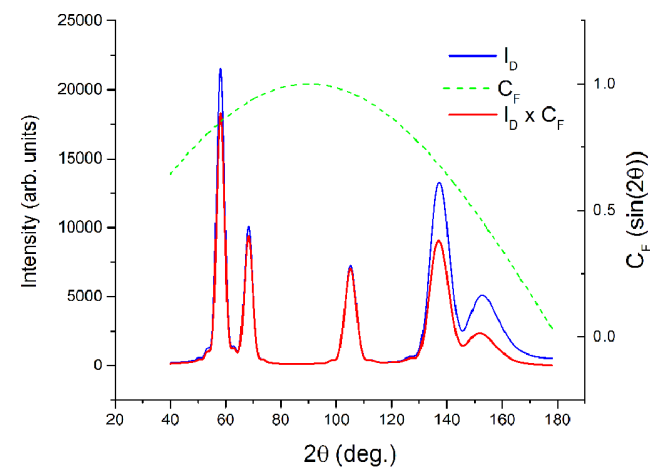

(a)

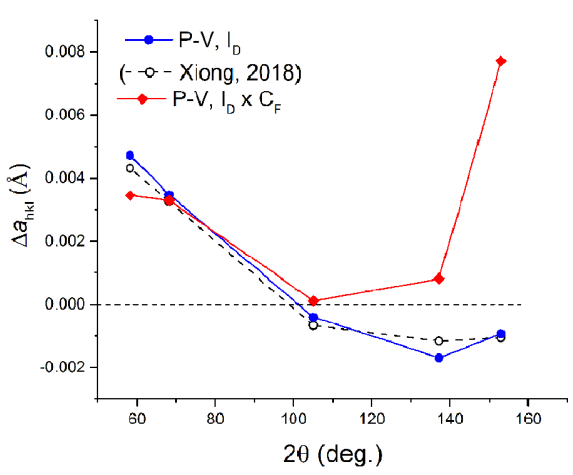

(b)
Figure 1

(a) Diffraction patterns for $5 \mathrm{~nm}$-diameter ideal Au spheroids, computed using the Debye formalism with $\mathrm{Cr} K \alpha$ radiation and after correction. The variation of the correction factor, $C_{\mathrm{F}}=\sin (2 \theta)$, over this angular range is also plotted. $(b)$ The deviation of the unit-cell parameters, $\Delta a_{h k l}$, from the ideal Au lattice parameter used in the simulation. Identical procedures utilizing pseudo-Voigt functions over identical ranges and background functions were used to fit both profiles. $\Delta a_{h k l}$ values reported in our original article are also included for reference (Xiong et al., 2018), since a different version of the fitting program, no longer available to us, was used at the time. 
article, and this exchange in Journal of Applied Crystallography, will stimulate the development of a rigorous theoretical framework for the analysis of nanocrystalline diffraction patterns. Until such a framework is in place, diffraction analysis results from such systems should be treated with caution given, also, that significant details of the distribution of unit-cell parameters within nanoparticles are eliminated during diffraction averaging (Xiong et al., 2019).

\section{References}

Kaszkur, Z. (2019). J. Appl. Cryst. 52, 693-694.

Öztürk, H., Yan, H., Hill, J. P. \& Noyan, I. C. (2015). J. Appl. Cryst. 48, 1212-1227.

Reynolds, R. C. (1986). Clays Clay Miner. 34, 359-367.

Xiong, S., Lee, S.-Y. \& Noyan, I. C. (2019). J. Appl. Cryst. 52, 262273.

Xiong, S., Öztürk, H., Lee, S.-Y., Mooney, P. M. \& Noyan, I. C. (2018). J. Appl. Cryst. 51, 1102-1115. 